Residence patterns and long-term movements of dolphins Tursiops truncatus, in the southeastern Gulf of Mexico

\title{
Patrones de residencia y movimientos a largo plazo de las toninas Tursiops truncatus, en la región sureste del Golfo de México
}

\author{
Alberto Delgado-Estrella ${ }^{1 *}$ \\ ${ }^{1}$ Facultad de Ciencias Naturales, Universidad Autónoma del Carmen. Calle 56 N 4, Ciudad del Carmen 24180, Campeche, México. \\ E-mail. delgadoestrella@gmail.com \\ ${ }^{*}$ Corresponding author
}

Introduction: There are scarce information about the movements and patterns of residence of bottlenose dolphins in the southern part of the Gulf of Mexico and the objective of this study is to have information on this subject, Terminos lagoon area is the zone with more effort to study in the region and it is possible that the dolphins move along the coast.

Methods: During 1994 to 1999 systematic surveys were carried out to study the movements and residence patterns of bottlenose dolphin populations in four different localities along the coast of the southern Gulf of Mexico, Holbox Island, Quintana Roo, Celestun in Yucatan, Terminos lagoon in Campeche and oriental Tabasco.

Results: In total, 2,889 dolphins were photo-identified in the four areas, the majority in the Terminos lagoon $(1,987)$; in this same area one dolphin was recorded within 10 years and nine months, thus having a multi-annual residency pattern. Other dolphin was photo-identified during 1997 in Holbox and 247 days later in Tabasco; another four animals moved from Terminos lagoon to Tabasco, travelling on average $270 \mathrm{~km}$.

Discussion and conclusions: These are the first long-term residence patterns and migration records for the species along the southern Gulf of Mexico. Bottlenose dolphins use the coastal lagoons as feeding and shelter areas for calving. The long movements recorded along the Yucatán peninsula between Quintana Roo and Tabasco may evidence a continuous coastal population along the southern Gulf of Mexico.

Key words: Bottlenose dolphins; Gulf of Mexico; movements; residence; Tursiops truncatus.

\section{Introducción}

En México los estudios poblacionales a largo plazo de mamíferos marinos son muy escasos, ya que la mayor parte de la información se conforma con las observaciones ocasionales de algunas especies en ciertas áreas de la Zona Económica Exclusiva de México. De las toninas o bufeos (Tursiops truncatus) se han estudiado algunas poblaciones, en las costas del Pacífico mexicano se han realizado algunos trabajos en Sinaloa (De la Parra-Venegas y Galván-Pastoriza 1985; Ortega-Ortiz y Delgado-Estrella 1996) y Sonora (Ballance 1990), en cuanto a abundancia, distribución y ciclos de actividad estacional y anual. Del Golfo de México existen informes de aspectos poblacionales para Veracruz (Heckel 1992; 
Morteo Ortiz et al. 2014), Tabasco (Delgado-Estrella y Pérez-Cortés 1993; López 1997) y Campeche (Delgado 2002; Bazúa-Durán y Delgado-Estrella 2014).

Se sabe que las toninas concentran sus actividades en ciertas áreas, conformando una o varias zonas de mayor actividad o áreas núcleo dentro de su ámbito hogareño, estas áreas pueden ser usadas estacionalmente por algunos individuos, o ser tomadas como sitios de residencia permanente por otros. El tamaño del ámbito hogareño puede estar en función de la densidad poblacional y también puede variar a lo largo del año, lo cual puede indicar una calidad ambiental variable (Shane et al. 1986).

Con el estudio de diversos grupos de toninas en diferentes regiones del sur de Golfo de México se pretende tener una mejor visión de cómo es su dinámica grupal, de sus movimientos, así como detectar cuáles son los factores que influyen para su congregación en estas zonas. Los objetivos de este trabajo fueron: 1) Utilizando la técnica de la fotoidentificación para detectar los movimientos más comunes en cuatro zonas de estudio en el sur del Golfo de México; 2) Conocer las variaciones en la residencia de las toninas y 3) Detectar movimientos a largo plazo entre las cuatro diferentes zonas de estudio.

\section{Material y métodos}

Áreas de estudio. Debido a las características y cercanía de las poblaciones de toninas, se planteó hacer la investigación en cuatro localidades a lo largo de la costa sur del Golfo de México y parte norte de la península de Yucatán (Figura 1). En Tabasco se seleccionó la franja costera comprendida entre la desembocadura del río Grijalva-Usumacinta en el poblado de Frontera, en la parte oriental $\left(18^{\circ} 37^{\prime} \mathrm{N},-92^{\circ} 40^{\prime} \mathrm{W}\right)$ y el poblado de Tupilco en la parte occidental $\left(18^{\circ} 36.30^{\prime} \mathrm{N}\right.$, $-93^{\circ} 20^{\prime} \mathrm{W}$ ).

La segunda localidad estudiada fue la laguna de Términos, Campeche, que se localiza entre los meridianos $-91^{\circ} 15^{\prime}$ y $-92^{\circ} 00^{\prime} \mathrm{W}$ y los paralelos $18^{\circ} 15^{\prime}$ y $19^{\circ} 00^{\prime} \mathrm{N}$, la cual tiene una longitud de $70 \mathrm{~km}$ y una anchura de $28 \mathrm{~km}$, estando limitada al norte por la isla del Carmen. Es una laguna somera, con una profundidad promedio de entre dos y cuatro metros, con excepción de las lagunas pantanosas de mareas y un canal profundo en la porción oriental de cada boca. La superficie aproximada de la cuenca principal es de $1,700 \mathrm{~km}^{2}$, pero incluyendo pantanos y sistemas fluvio-lagunares asociados al área, llega aproximadamente a 2,500 km² (Yáñez-Arancibia et al. 1988).

La tercera zona, en el estado de Yucatán, fue las cercanías del poblado de Celestún. Esta laguna o ría, como también se le conoce, se localiza entre los paralelos $20^{\circ} 48^{\prime}$ y $20^{\circ} 58^{\prime} \mathrm{N}$ y los meridianos $-90^{\circ} 15^{\prime}$ y $-90^{\circ} 25^{\prime} \mathrm{W}$. Tiene una extensión aproximada de $3 \mathrm{~km}^{2}$. La vegetación de la región es de tipo matorral espinoso, con plantas carnosas y pastizal. Está rodeada de manglar tipo borde, dominado por Rhizophora mangle. La vegetación subacuática está constituida principalmente por Chara fibrosa y Bathophora oerstendi en la porción norte. En la porción centro y sur las especies que sobresalen son: Diplantera (Halodule) wrightii, Syringodium filiforme y el alga Chaetomorpha linum, a las cuales se les encuentra en los márgenes de la laguna, formando densas alfombras (Herrera-Silveira 1990).

La cuarta zona correspondió a la laguna de Yalahau, que se localiza en el estado de Quintana Roo, entre los paralelos $21^{\circ} 26^{\prime}$ y $21^{\circ} 36^{\prime} \mathrm{N}$ y los meridianos $-87^{\circ} 08^{\prime}$ y $-87^{\circ} 29^{\prime} \mathrm{W}$. También se le conoce como laguna Conil y limita al Golfo de México a través de la isla Holbox. Toda esta región sureste del Golfo de México presenta un clima cálido subhúmedo con lluvias en verano (Aw1 $\left.\left(x^{\prime}\right)\left(i^{\prime}\right)\right)$. La temperatura promedio anual es de $26.1^{\circ} \mathrm{C}$, los meses más calientes son de mayo a septiembre. La precipitación promedio anual es de 1,290 mm, siendo los meses más lluviosos de junio a septiembre (García 1973). En la región se presentan tres temporadas climáticas, que 
son: secas (febrero a mayo), lluvias (junio a septiembre) y nortes (octubre a enero). Los vientos dominantes son del sureste, de marzo a septiembre. En el verano y en el otoño se presentan las "turbonadas", o tormentas tropicales aisladas y rápidas.

El comportamiento de los nutrientes en el Golfo de México responde, al igual que el oxígeno disuelto, a los distintos eventos de circulación que están influenciados por la estacionalidad y, consecuentemente, al ascenso de aguas del fondo por enfriamiento de masas, giros ciclónicos, surgencias y por hundimientos anticiclónicos (De la Lanza Espino 1991).

En cuanto a las características hidrográficas de esta parte del Golfo de México, el flujo de agua a través del canal de Yucatán, a lo largo del margen noreste del banco de Campeche, forma un verdadero frente: la corriente del Lazo, que corre hacia el norte del Golfo de México sobre aguas profundas. En este punto el flujo es inestable y se presenta un giro anticiclónico en la parte este del Golfo, pasando del banco de Campeche al borde oeste de la península de Florida. La corriente del Lazo penetra hacia el oeste del Golfo cuando se separa del banco de Campeche (Mollinari y Morrison 1988).

En el Golfo de México se presenta una capa superficial de baja salinidad con una capa de mezcla de hasta $50 \mathrm{~m}$ en la corriente de Lazo. Durante el verano la capa de mezcla es muy superficial, variando de 10 a 20 m (Longhurst 1998). La clorofila en esta región tiene variaciones características, por la entrada de agua costera a la zona pelágica y la surgencia de nutrientes (Paluskiewicz et al. 1983), mientras que los giros anticiclónicos modifican significativamente la distribución de nutrientes en la parte oeste. Los remolinos anticiclónicos y la corriente presentan una topografía de la picnoclina en forma de domo, en la que los nitratos suben a la zona fótica alrededor de su perímetro (Walsh et al. 1989).

Se realizaron salidas periódicas en cada zona en un periodo de cinco años, abarcando cada una de las temporadas climáticas. La zona mejor estudiada fue la laguna de Términos y la menos

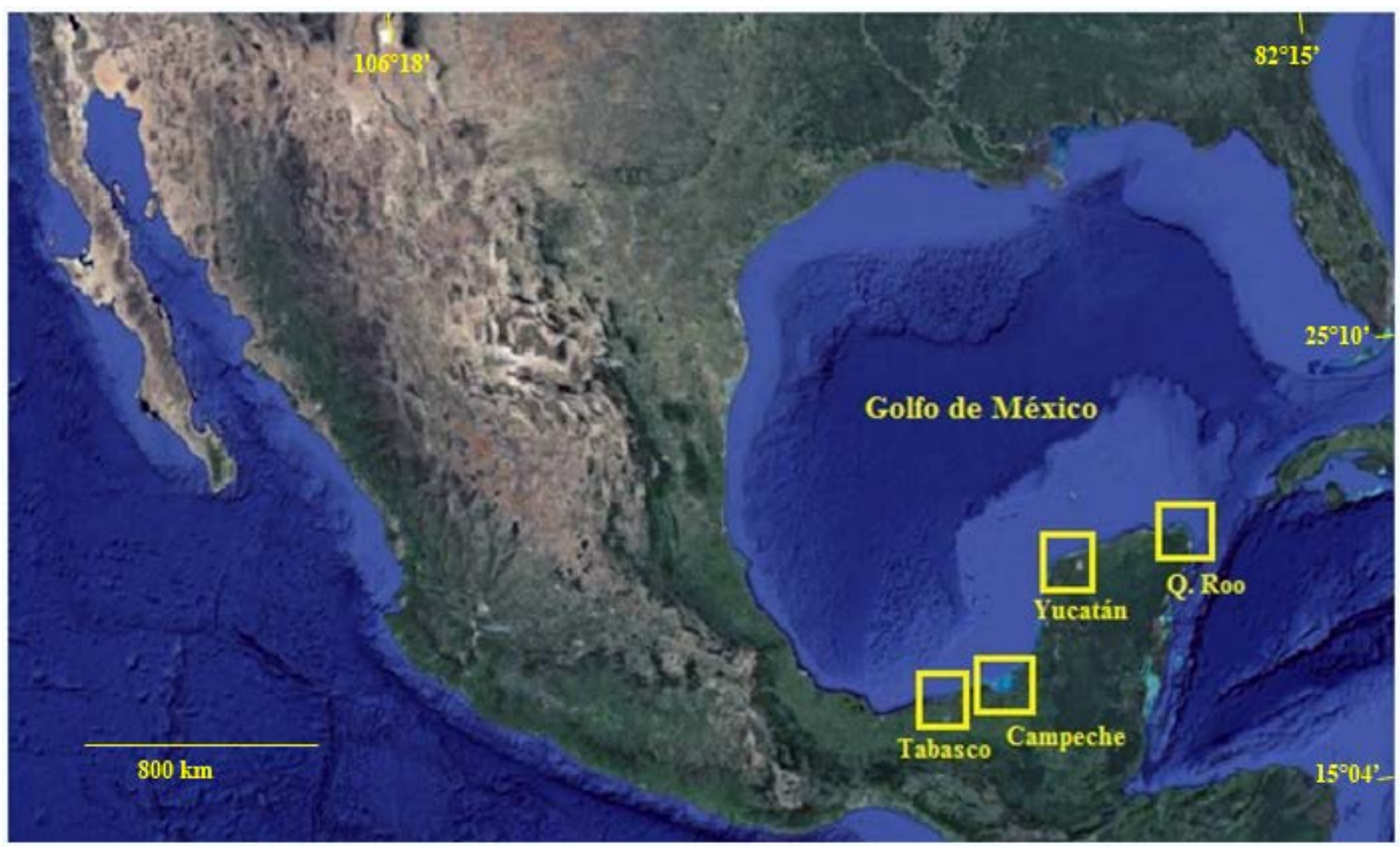

Figura 1. Localización de áreas de estudio en el sureste del Golfo de México (Tomado de Google Earth, 15 abril, 2015, y modificado por el autor). 
estudiada la zona de Celestún. Se utilizaron lanchas de fibra de vidrio de siete metros de eslora, con motor fuera de borda de entre 55 y $75 \mathrm{HP}$.

En cada avistamiento se fotografiaron las aletas dorsales de los animales utilizando al menos dos cámaras tipo "reflex", equipadas con lentes de acercamiento de entre 210 y 300 mm. Se utilizó película blanco y negro (ASA 800).

En cuanto al análisis de fotoidentificación, se realizaron algunas modificaciones al método descrito por Würsig y Jefferson (1990) y Defran et al. (1990), debido a que la comparación de las aletas dorsales entre los diferentes individuos fotografiados en cada salida de cada zona estudiada se realizó con base en trazados en hojas de papel. Todas las aletas se imprimieron en un tamaño estándar, para su revisión y comparación (Delgado 2002). Para mantener un control sobre los trazos y evitar sesgos de la persona que trazaba, se comparó cada dibujo con la fotografía original, para hacer las correcciones pertinentes o bien repetir de nuevo el trazo. Para las comparaciones se realizó una selección preliminar de las características en cuanto a la forma y número de muescas de las aletas, estableciendo las siguientes categorías: una muesca, dos muescas, tres muescas, más de tres muescas, muescas en la punta, muescas en el borde anterior de la aleta, aletas con más de tres muescas, incluyendo la punta.

Primero se elaboraron los catálogos fotográficos de cada zona, detectando los individuos con al menos un reavistamiento. Posteriormente se comparon los individuos entre la diferentes zonas de estudio, confirmando los posibles reavistamientos directamente con las fotografías. Solamente se utilizaron imágenes con buena calidad, de tal modo que se pudieran diferenciar las muescas y marcas distintivas en cada ejemplar (Delgado 2002). Para establecer los patrones de residencia se tomaron en cuenta los criterios de Ballance (1990), considerándose como no residentes a las toninas que fueron observadas una sola vez y como residentes a las toninas que se observaron más de tres veces (recapturas), tomando en cuenta también las temporadas en que fueron registradas.

\section{Resultados}

La laguna de Términos en Campeche fue la zona en donde se pudo fotoidentificar el mayor número de individuos y la zona con menor número de toninas identificadas fue Celestún, en Yucatán. En la Tabla 1 se muestran los totales de toninas identificadas por zona de estudio y la proporción que representa del número total de ejemplares. En las cuatro zonas de estudio se obtuvieron reavistamientos, algunos con unos cuantos minutos de diferencia y otros más con periodos tan largos como 11 años. También se reavistaron individuos en zonas comunes, y hubo animales que siempre fueron localizados en lugares diferentes y distantes entre sí.

En la costa de Tabasco, 20 de las 521 toninas identificadas pudieron ser reavistadas (3.8 \%), la mayoría de ellas durante el mismo año o en años consecutivos, y solo una con más de cuatro años de diferencia entre la captura y la recaptura fotográfica (TTTA-060). Sólo un individuo (TTTA-084) se reavistó dos veces, primero durante el mismo mes y después a casi dos meses. La diferencia más grande en distancia entre un avistamiento y otro, independientemente del tiempo transcurrido, fue de $44.4 \mathrm{~km}$. En individuos reavistados el mismo día se pudo calcular la velocidad de movimiento y desplazamientos diarios. En Tabasco se registraron dos de estos casos 43.6 $\mathrm{km} / \mathrm{h}$ y $6.4 \mathrm{~km} / \mathrm{h}$.

La mayor parte de los reavistamientos se registraron en las entradas a las lagunas costeras y ríos como el González y el Grijalva. En esta parte occidental de la costa de Tabasco fue común encontrar a las toninas asociadas a barcos camaroneros trabajando, entre 30 y $50 \%$ de los avistamientos estuvieron asociados con esta actividad pesquera. De igual manera, los avistamientos registrados en la parte central de la costa de Tabasco estuvieron asociados con 
actividades pesqueras, ya sea con redes de cerco o redes agalleras, y con la pesquería de la sierra (Scomberomorus spp.) y el bonito (Sarda sarda).

En la laguna de Términos, Campeche, se registraron los movimientos más interesantes y se pudo hacer un mejor seguimiento, ya que hubo mayor número de reavistamientos de los individuos identificados. En la Figura 2 se señalan los movimientos diarios más comunes en la boca del Carmen. En esta parte de la laguna se registró la mayoría de las toninas identificadas.

Para fines prácticos solo se describirán los movimientos de los animales identificados considerados más importantes, ya sea por el tiempo transcurrido entre los registros, por las distancias recorridas o bien por la asociación con otros individuos. Se registraron delfines que no se movieron mucho de su zona habitual de acción durante los diferentes registros. El caso más interesante fue el del ejemplar identificado inicialmente con el número TTLT-001 ("Mocho"), que fue registrado de manera regular durante más de 10 años en la zona de la boca del Carmen y parte occidental de la laguna.

Debido al gran número de individuos con reavistamientos en zonas distantes o con mucho tiempo entre capturas (más de 3 años), se elaboraron mapas de localización señalando algunos ejemplos representativos de los individuos en la laguna de Términos. En el interior de la laguna de Términos se registraron movimientos tanto de corta duración y distancia, menos de $10 \mathrm{~km}$ en pocos minutos, así como movimientos intermedios de 20 a $45 \mathrm{~km}$. Los recorridos más largos los realizaron las toninas que viajaron de una boca a otra y que, dependiendo de la ruta, recorrieron más de 60 km (Figura 2). En la cuenca de la laguna se observó una gran variedad de movimientos. Seis de las toninas identificadas y con reavistamientos (individuos TTLT-052, 56, 174, 178, 1828 y 1833) se localizaron en ambas bocas de la laguna en tiempos diferentes, promediando una distancia entre puntos de $46.3 \pm 2 \mathrm{~km}$.

Otras toninas se movieron principalmente en la parte sur de la laguna, viajando frecuentemente de y hacia la laguna de Panlao, mientras que otros individuos se movieron con frecuencia de la boca del Carmen a lo largo del litoral interno de la isla. Dos individuos (TTLT-470 y 1263) se localizaron por separado en la boca del Carmen en años diferentes, y durante 1998 se vieron juntos en el Golfo de México, frente a la isla del Carmen.

La fidelidad al sitio varió entre los individuos, ya que algunos se movieron mucho mientras que otros siempre se localizaron en la misma zona; por ejemplo, el individuo TTLT-114 de las nueve ocasiones que se observó, en las primeras cuatro se localizó entre cinco y $10 \mathrm{~km}$ de la laguna de Panlao y las últimas seis veces siempre se localizó en el interior de Panlao; algo similar sucedió

Tabla 1. Número de toninas Tursiops truncatus, totales identificadas en las diferentes localidades de estudio de la región sureste del Golfo de México.

\begin{tabular}{|c|c|c|c|c|}
\hline Localidad & Periodo de estudio & $\begin{array}{l}\text { Número total de individuos } \\
\text { fotoidentificados }\end{array}$ & $\begin{array}{l}\text { Número de } \\
\text { individuos } \\
\text { recapturados }\end{array}$ & $\begin{array}{c}\% \text { de } \\
\text { recaptura }\end{array}$ \\
\hline Laguna de Términos, Campeche & $\begin{array}{c}\text { 1989-90; 1995-1998; } 1999 \\
\text { (6 años de esfuerzo, } 10 \text { años } \\
\text { totales) }\end{array}$ & $\begin{array}{c}1987 \\
(68.7 \%)\end{array}$ & $\begin{array}{c}155 \\
(80.8 \%)\end{array}$ & 7.8 \\
\hline Isla Holbox, Q. Roo & $\begin{array}{l}\text { 1994-1995, 1996-1998 (3 } \\
\text { años de esfuerzo, } 4 \text { años } \\
\text { totales) }\end{array}$ & $\begin{array}{c}344 \\
(11.9 \%)\end{array}$ & $\begin{array}{c}13 \\
(6.7 \%)\end{array}$ & 3.8 \\
\hline Costa de Tabasco & $\begin{array}{c}\text { 1992, 1996, 1998-1999 (3 } \\
\text { años de esfuerzo, } 7 \text { años } \\
\text { totales) }\end{array}$ & $\begin{array}{c}521 \\
(18.0 \%)\end{array}$ & $\begin{array}{c}20 \\
(10.4 \%)\end{array}$ & 3.65 \\
\hline Celestún, Yucatán & $\begin{array}{l}\text { 1997-1998 (un año de } \\
\text { esfuerzo total) }\end{array}$ & $\begin{array}{c}37 \\
(1.3 \%)\end{array}$ & $\begin{array}{c}4 \\
(2.1 \%)\end{array}$ & 10.8 \\
\hline & Totales & 2,889 & 192 & 6.6 \\
\hline
\end{tabular}

Nota: la primera parte se refiere al esfuerzo de fotoidentificación y el esfuerzo total incluye esfuerzo en el que no se obtuvieron fotos. 
con el individuo 314, que al menos en cuatro ocasiones se vio en la misma manada, junto con el individuo 114. Otros ocho individuos únicamente fueron localizados en el interior de Panlao realizando diversas actividades y en sus grupos regularmente se presentaron crías, algunas de ellas recién nacidas.

Se registraron dos movimientos con dos días de diferencia, los cuales se movieron 37 y 45 $\mathrm{km}$ respectivamente. Los individuos con número 1673, 1709, 1710 y 1711 formaron un "grupo núcleo" de un día a otro, viajando $32 \mathrm{~km}$ desde la laguna de Panlao a la parte noreste de la isla del Carmen, llamándole "grupo núcleo" porque hubieron otros individuos asociados a las manadas que ya no aparecieron integrados en ninguno de los dos días consecutivos de avistamientos.

En la costa de Yucatán únicamente cuatro individuos fueron reavistados, todos ellos muy cercanos entre sí. El individuo TTCE-001 se registró tres días consecutivos y la distancia entre los puntos más distantes fue de $12 \mathrm{~km}$. El único delfín que se registró con tres meses de diferencia fue el individuo TTCE-005, con una diferencia de $18 \mathrm{~km}$ entre ambos puntos. Todos los avistamientos se registraron entre la localidad de Celestún y el Palmar. La máxima diferencia registrada en una tonina identificada entre capturas consecutivas fue de 90 días, es decir no hubo ningún individuo que se identificara en salidas consecutivas, por lo cual no se pudo establecer ningún tipo de residencia, y al parecer los animales se mueven a lo largo de la costa en una zona mucho más grande que la estudiada.

La zona de isla Holbox presentó 14 casos de reavistamientos. Seis individuos solo presentaron un reavistamiento, cuatro de ellos en el mismo año y los restantes en años diferentes, pero en zonas muy cercanas, con excepción de un individuo que primero se localizó en la laguna de Yalahau y posteriormente tres años después se observó en el Golfo de México. Seis ejemplares presentaron dos recapturas, la más interesante fue de los individuos TTIH-037 y 044, que se vieron

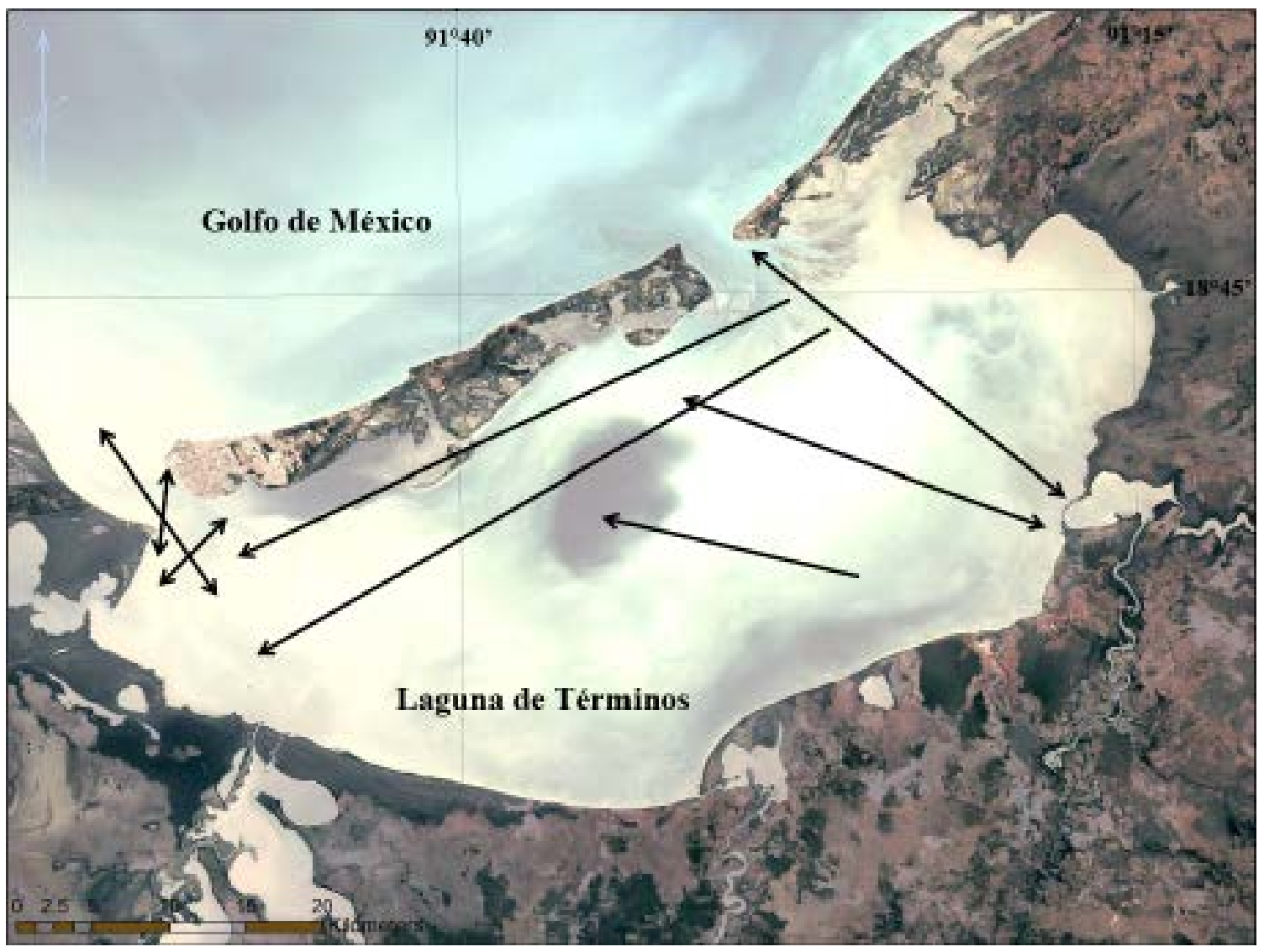

Figura 2. Movimientos más comunes de las toninas en la laguna de Términos, Campeche (Tomado de Google Earth, 15 abril, 2015, y modificado por el autor). 
juntos primero en la laguna de Yalahau y posteriormente se recapturaron en el Golfo de México, formando parte de grupos diferentes.

Solo dos toninas se recapturaron tres veces, TTIH-007 y 089, todas ellas en el interior de la laguna de Yalahau, entre 1994 y 1997; la última vez ambas se encontraron en la misma manada. La distancia recorrida en un solo día fue de $4.5 \mathrm{~km}$, con una diferencia de 74 minutos, dando una velocidad promedio de movimiento de $3.65 \mathrm{~km} / \mathrm{h}$. La diferencia más grande en distancia entre avistamientos, sin tomar en cuenta el tiempo transcurrido, la presentó el individuo TTIH-044, con 35.5 km.

Existieron movimientos a largo plazo realizados por algunos de los individuos fotoidentificados en el Golfo de México. Se pudieron diferenciar cinco toninas con reavistamientos muy distantes de la primera localidad registrada, en la Tabla 2 se resumen los datos de cada una. Un individuo (TTIH-232) se movió de la zona de isla Holbox a la costa de Tabasco en menos de un año, recorriendo al menos 800 km (Figura 3). Los otros cuatro individuos se movieron entre la laguna de Términos, Campeche y la costa de Tabasco siempre en este orden, ya que primero fueron catalogados en Campeche y posteriormente en Tabasco (Fig. 4), recorriendo una distancia promedio de $270 \mathrm{~km}$ ( $\pm 36.6 \mathrm{~km}$ ).

Los resultados tanto de la costa de Tabasco como de Celestún, Yucatán, no aportaron información suficiente para determinar algún tipo de residencia, y tal parece que las toninas se mueven continuamente a lo largo de la costa. En contraste, las zonas de lagunas costeras sí mostraron patrones de residencia bien definidos. Estos patrones se apoyan en los reavistamientos de un estado a otro y con las fluctuaciones en los valores de abundancia estacional.

En la laguna de Yalahau, Quintana Roo, al menos cuatro individuos (TTIH-007, TTIH-043, TTIH055 y TTIH-089) fueron observados siempre en la laguna entre 1994 y 1997, por lo que pueden considerarse como residentes multianuales. Por otro lado, el individuo TTIH-037 y el TTIH-044 primero se observaron casi frente al poblado de isla Hobox y en el interior de la laguna respectivamente, en julio de 1994 y posteriormente se localizaron en las inmediaciones de Cabo Catoche en el Golfo de México durante 1994 y 1995, por lo que pueden considerarse residentes anuales con mayor área de movimiento. Cinco de los individuos identificados se observaron durante 1994 y posteriormente se recapturaron en julio de 1997, por lo que se podrían considerar como visitantes ocasionales.

En la laguna de Términos se tuvo el caso de la única tonina que se registró en una misma zona durante más de 10 años. Por otra parte, un grupo de toninas que presentaron mutilaciones en la cola y que frecuentaron la boca de Puerto Real durante 1989-1990, se volvieron a recapturar durante 1995, 1996 y 1997, siendo casos de residencia multianual en zonas específicas como las bocas de

Tabla 2. Información de individuos reavistados en localidades diferentes del Golfo de México.

Primer avistamiento

\begin{tabular}{|c|c|c|c|c|c|c|}
\hline $\mathbf{N}^{\circ}$ Catálogo & Fecha & Localidad & № Catálogo & Fecha & Localidad & $\begin{array}{l}\text { Tiempo transcurrido } \\
\text { Distancia recorrida }\end{array}$ \\
\hline TTIH-232 & $15 / 11 / 95$ & $\begin{array}{l}\text { Punta Caracol, Quintana } \\
\text { Roo }\end{array}$ & TTTA-142 & $20 / 7 / 96$ & 5.5 E Chiltepec, Tabasco. & $\begin{array}{l}247 \text { días } \\
800 \text { km }\end{array}$ \\
\hline TTLT-056 & $17 / 8 / 95$ & 7.5 NW Panlao, Campeche & TTTA-460 & $26 / 6 / 99$ & $\begin{array}{c}18^{\circ} 31.26 \mathrm{~N}-93^{\circ} 15.58 \mathrm{~W} \\
\text { Tabasco }\end{array}$ & $\begin{array}{l}\text { 1,404 días } \\
320 \mathrm{~km}\end{array}$ \\
\hline TTLT-183 & $23 / 12 / 95$ & 10 SW Panlao, Campeche & TTTA-423 & $16 / 6 / 99$ & $\begin{array}{c}18^{\circ} 28.00 \mathrm{~N}-93^{\circ} 13.20 \mathrm{~W} \\
\text { Tabasco }\end{array}$ & $\begin{array}{l}\text { 1,264 días } \\
250 \mathrm{~km}\end{array}$ \\
\hline TTLT-428 & 29/3/96 & $0.5 \mathrm{~S}$ Zacatal, Campeche & TTTA-454 & 26/6/99 & $\begin{array}{c}18^{\circ} 31.26 \mathrm{~N}-93^{\circ} 15.58 \mathrm{~W} \\
\text { Tabasco }\end{array}$ & $\begin{array}{l}\text { 1,176 días } \\
240 \text { km }\end{array}$ \\
\hline TTLT-1486 & 9/10/97 & $\begin{array}{l}2 \text { NW Balchacah, } \\
\text { Campeche }\end{array}$ & TTTA-297 & 28/3/99 & $\begin{array}{c}18^{\circ} 30.44 \mathrm{~N}-93^{\circ} 06.28 \mathrm{~W} \\
\text { Tabasco }\end{array}$ & $\begin{array}{l}535 \text { días } \\
270 \mathrm{~km}\end{array}$ \\
\hline
\end{tabular}

Reavistamiento 
la laguna. Al menos tres individuos fueron localizados en la laguna de Términos, Campeche, y posteriormente en la costa de Tabasco (Figura 4), al parecer esta es una zona con intercambio de individuos por su cercanía.

El promedio general de eficiencia fotográfica (esto es, el número de toninas fotoidentificadas del total de animales observados en cada manada) durante todo el estudio y en todas las zonas fue de alrededor de $50 \%$. Cabe señalar que la eficiencia tuvo una variación dependiendo de la zona de estudio, de las condiciones ambientales y del comportamiento de las toninas, ya que varió desde 10 hasta $100 \%$ en varios de los grupos. Coincidentemente en todas las zonas, las eficiencias menores se presentaron en la temporada de nortes o bien en días con mucha marejada o lluvia; en este último caso, aunque las toninas estuvieran cerca de la lancha y no se movieran mucho, no se pudieron utilizar las cámaras fotográficas.

\section{Discusión}

Los estudios de identificación de individuos pueden ser usados para determinar patrones de movimiento y de asociación entre animales (Delgado 2002). Con base en los reavistamientos de los individuos conocidos, se ha determinado que algunos animales regresan consistentemente a una localidad particular y otros pueden mostrar especificidad temporal, como regresar a ciertas localidades en la misma temporada. Los individuos de diferentes clases de edad y sexo, con frecuencia muestran diferencias en la fidelidad al sitio o pueden presentar diferencias estacionales o temporales (Hammond et al. 1990).

No necesariamente debe de existir una relación directa entre los valores de abundancia de toninas en una determinada zona de estudio, con la cantidad de individuos que pueden ser identificados con fotografía, ya que hay factores que afectan la cantidad y calidad de las imágenes obtenidas, por ejemplo: la accesibilidad a los delfines, tipo de conducta que están realizando, condiciones ambientales como el oleaje, viento y corrientes, condiciones de luminosidad, experiencia y destreza del fotógrafo, tipo de película fotográfica y equipo fotográfico utilizado, entre otros.

En este caso, sí hubo correspondencia entre el número de individuos identificados con las abundancias relativas registradas en cada zona (Delgado 2002), aunque hubo zonas como Celestún en donde resultó más fácil obtener las fotografías de las aletas dorsales de las toninas. El segundo lugar más fácil para fotografiar a las toninas fue la laguna de Términos, seguida de los alrededores de isla Holbox y por último la costa de Tabasco, en donde por circunstancias tales como la captura de animales vivos para delfinarios, así como la cacería por parte de los pescadores locales para utilizar su carne, los delfines se ahuyentan fácilmente con la presencia de las lanchas, por lo que no se pudieron obtener buenas fotos en algunas de las manadas observadas.

Otro factor común fue que en determinadas temporadas los incrementos de individuos nuevos son muy grandes, y coinciden también en todas las zonas con el inicio de la temporada de lluvias durante el mes de julio. Esto también se reflejó en una mayor incidencia de avistamientos durante la temporada de lluvias en prácticamente todas las zonas, marcando un periodo de migración local de zonas aledañas, o bien de individuos que se mueven varios cientos de kilómetros.

El gran número de toninas identificadas en la laguna de Términos (casi 2,000) es muy grande como para que todas convivan en la misma zona al mismo tiempo, y más aún que el hábitat resista tal presión ambiental. Esta condición se refuerza la hipótesis de que hay flujos intermitentes de toninas que vienen tanto de zonas costeras cercanas como las que viven mar adentro, y es variable a lo largo del año en intensidad y duración, utilizándose la zona preferentemente por hembras con crías (Delgado 2002). Las estimación poblacional para la laguna de Términos en una sola temporada fue de 560 a 940 individuos (Delgado et al. 1994), pero podrían superar los 
1,000 individuos si tomamos en cuenta los animales que se distribuyen frente a la costa de la isla del Carmen, y el valor de 1,987 debe tomarse como el total de individuos identificados a lo largo de 10 años de trabajo.

Bräger et al. (1994), aplicando la técnica de fotoidentificación en la bahía de Galveston, Texas durante 1990-1991, identificaron más de 1,000 individuos; la mayor parte de estos individuos fotoidentificados solo se observaron una vez y se concluyó que la mayoría de los delfines únicamente transitan por la zona y solo un núcleo de cerca de 200 individuos usaron el área durante mucho tiempo. Al respecto en la laguna de Términos, menos de un centenar de toninas se identificaron de forma constante como para considerarlas residentes multianuales.

Hasta donde se sabe, en la costa de Yucatán es la primera vez que se realiza esfuerzo de fotoidentificación. Bearzi (1996) observó en el Palmar 28 avistamientos, mientras que en Ría Lagartos avistaron 47 grupos en 14 y 11 horas de observación, respectivamente.

Con excepción de la laguna de Términos, en donde hubo un individuo que presentó más de 40 recapturas, en las zonas restantes la mayoría de los individuos con reavistamientos tuvieron únicamente dos recapturas y dos individuos en isla Holbox presentaron tres. Dependiendo de la zona, entre 45 y $95 \%$ de los individuos tuvieron solo una captura fotográfica.

Por ejemplo, en la laguna de Términos menos de $8 \%$ de las toninas identificadas presentaron recapturas, pero este valor es elevado comparado con los valores de menos de $4 \%$ registrados tanto en las demás zonas de estudio como lo registrado en otros trabajos en el estado de Quintana Roo (Zacarías y Zárate 1992; Lechuga et al. 1995). El valor registrado en Celestún de 10.8 \% está sesgado por el número tan reducido de animales identificados. De igual manera, el valor general de $6.6 \%$ de individuos con recapturas es adecuado, tomando en cuenta los antecedentes señalados.

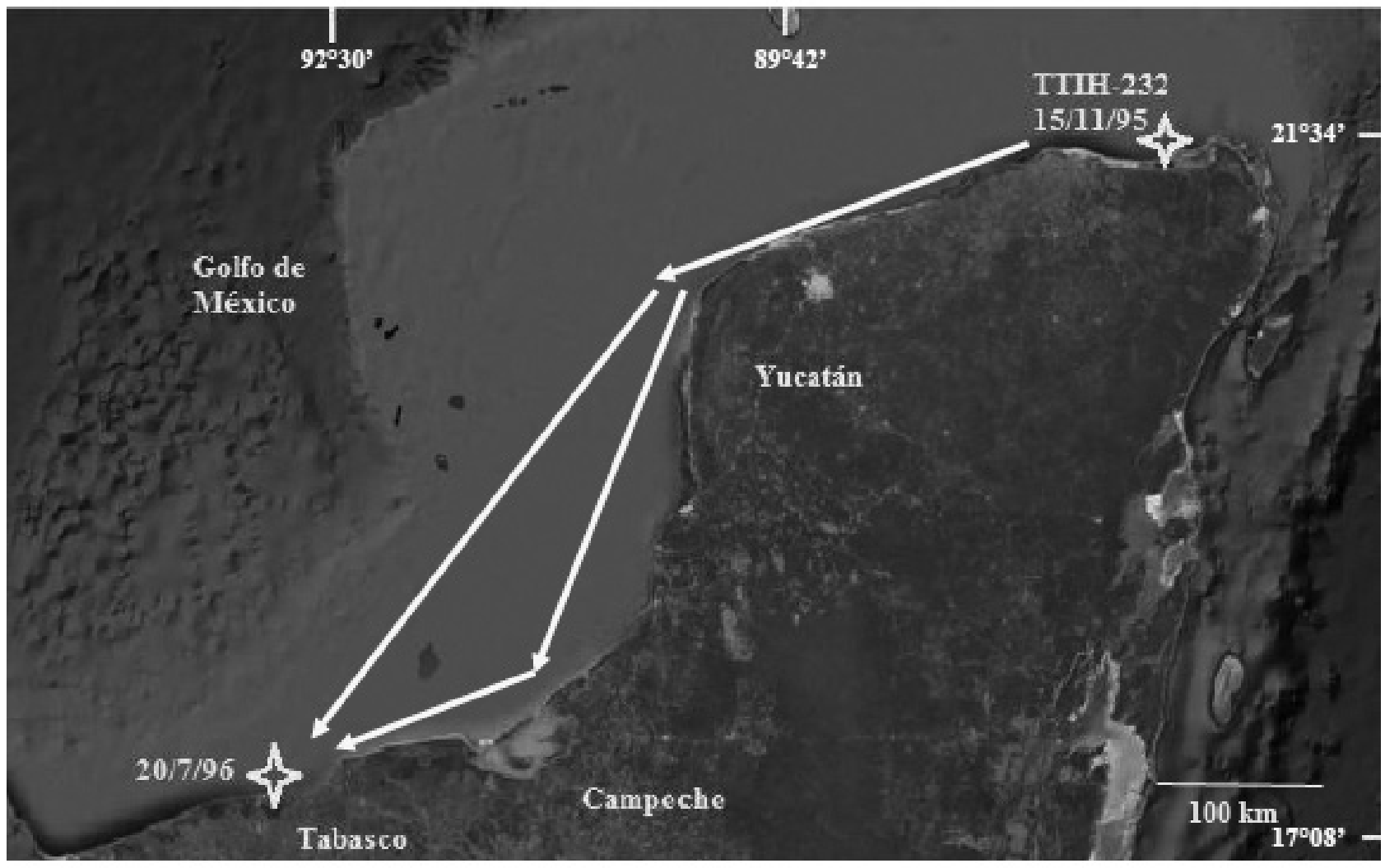

Figura 3. Movimiento realizado por la tonina fotoidentificada por primera vez en la costa de Quintana Roo y posteriormente reavistada en la costa de Tabasco (Tomado de Google Earth, 15 abril, 2015, y modificado por el autor). 
Hubo individuos que, gracias a lo conspicuo de sus marcas en las aletas dorsal o caudal, pudieron ser identificados de manera visual aunque no se fotografiaran, mientras que otros individuos solo pudieron diferenciarse al analizar las fotografías, identificando y clasificando pequeñas marcas en sus aletas dorsales; éstos pudieron utilizarse para tener datos de presencia y utilización de las diferentes áreas en la laguna de Términos.

En cuanto al cambio de marcas en la aleta dorsal, en este trabajo únicamente un individuo identificado en la laguna de Términos presentó cambios en su aleta, incrementando con una muesca su contorno original, pero gracias al patrón de marcas y a la posición de las mismas pudo ser identificado. Otros cambios observados involucraron otras partes del cuerpo, sobre todo algunas cicatrices de mordidas de otros individuos, pero estas marcas no fueron permanentes y no son útiles para fines de identificación a largo plazo. Al respecto, Darby et al. (1995) señalan que la frecuencia de cambio de las cicatrices en las aletas dorsales de las toninas es mayor en machos subadultos $(50 \%)$ y menor en hembras subadultas (11\%). La mayor propensión que tienen los machos adultos para presentar heridas puede estar relacionada con la competencia por las hembras, disputas por territorio, mayor vulnerabilidad hacia los depredadores como consecuencia de tamaños de grupo menores o la combinación de todos los factores (Wells et al. 1987). Los cambios en la longitud de la aleta dorsal sugieren que se presentan cuando el animal es joven, ya que cuando es adulto el cambio es mínimo. Las diferencias en las marcas naturales entre los sexos y las clases de edad refuerzan la idea de que pudieran existir diferencias en la estructura social con respecto a la edad y al sexo.

En vida libre las toninas jóvenes están expuestas a un mayor grado de interacción con sus congéneres, lo que representa mayor probabilidad de infringirse heridas en cualquier parte de su cuerpo; también es común la interacción con embarcaciones y redes de pesca, lo cual les ocasiona lesiones diversas. Cuando un delfín se enreda en una línea de pesca puede causarle la muerte, o liberarse con cortaduras que le infringen heridas principalmente en los apéndices, o incluso quedar parcial o totalmente cercenados. Sobre todo en la costa de Tabasco y en Campeche se registraron varios individuos con la aleta dorsal prácticamente cortada a la mitad o sin punta y un individuo en la boca del Carmen presentó prácticamente cercenado el pedúnculo caudal, quedando la columna vertebral desviada, siendo evidente al salir a respirar.

Se registró una gran gama de movimientos entre las toninas identificadas, dependiendo del tipo de hábitat, ya que algunas de las toninas que habitaron zonas de lagunas costeras utilizaron tanto la zona costera como la cuenca lagunar, y algunas otras se observaron predominante o únicamente en el interior de las lagunas.

Casi ninguno de los individuos que presentaron al menos una recaptura estuvieron formando parte del mismo grupo, el común denominador fue que siempre hubo cambio de integrantes en la manada, incluso cuando la diferencia entre el avistamiento fuera de unos pocos minutos. Sólo se presentaron dos casos en que más de dos individuos conservaron su agrupación y en otras dos ocasiones solo parejas se conservaron juntas, esto último en la laguna de Términos, Campeche.

Una de las excepciones encontrada fue en la costa de Tabasco, en donde cuatro individuos identificados dos veces en el mismo día con 11 minutos de diferencia, siguieron juntos a ocho kilómetros de distancia del primer punto de registro. Sin embargo, las asociaciones temporales de este tipo pueden durar horas, días o semanas. La mayoría de los reavistamientos se dieron con pocos meses de diferencia y algunos de un año a otro, sin poderse establecer un patrón de residencia definido, considerándose a las toninas como visitantes ocasionales o estacionales que llegan a la zona para alimentarse cuando hay abundancia de presas, y posteriormente pueden presentar o no actividad social con los otros individuos.

En la laguna de Términos se presentaron muchas variantes en los movimientos de las toninas, 
ya que, por ejemplo, en ambas bocas lo más común fue que los animales entraran y salieran de la laguna utilizando el canal que está ubicado en la parte oriental. Esto podría estar relacionado con que los animales utilizan de manera preferencial las diferentes zonas para realizar sus actividades, dependiendo de las temporadas y la hora del día.

El canal de la boca de Puerto Real fue la única zona que se usó como acceso y salida, mientras que en la boca del Carmen se registraron toninas a todo lo ancho de la boca. Ambas bocas presentan puentes en la actualidad, pero hasta antes de 1995 únicamente la boca de Puerto Real tenía esta estructura. Aparentemente la presencia del puente en la boca del Carmen no cambió los patrones y rutas de movimiento en esta zona, ya que comparando los avistamientos registrados antes de 1995 y posteriores a la construcción del puente, tienen una distribución similar. El patrón de movimientos de los delfines para entrar y salir a la laguna son prácticamente los mismos que entre 1989 y 1990 (Delgado 1991).

El caso de residencia más interesante de todo el trabajo fue el primer individuo identificado en la laguna de Términos durante marzo de 1989 (TTLT-001 “Mocho"). El cual permaneció siempre en esta boca y en general en la parte occidental de la laguna, la parte más al este que se le registró fue entre la zona de boca Chica y la desembocadura de la laguna de Balchacah, al sureste de la boca del Carmen, casi a 33 km de distancia en línea recta de este punto.

En la boca de Puerto Real se registró un grupo de cinco toninas que tuvieron diferentes grados de mutilación en la aleta caudal y siempre fueron localizados en esta boca o en sus cercanías, es decir en el delta interno. Todos estos individuos se identificaron por primera vez en 1989 y se fueron registrando a lo largo del estudio de manera irregular. Únicamente uno de ellos presentó

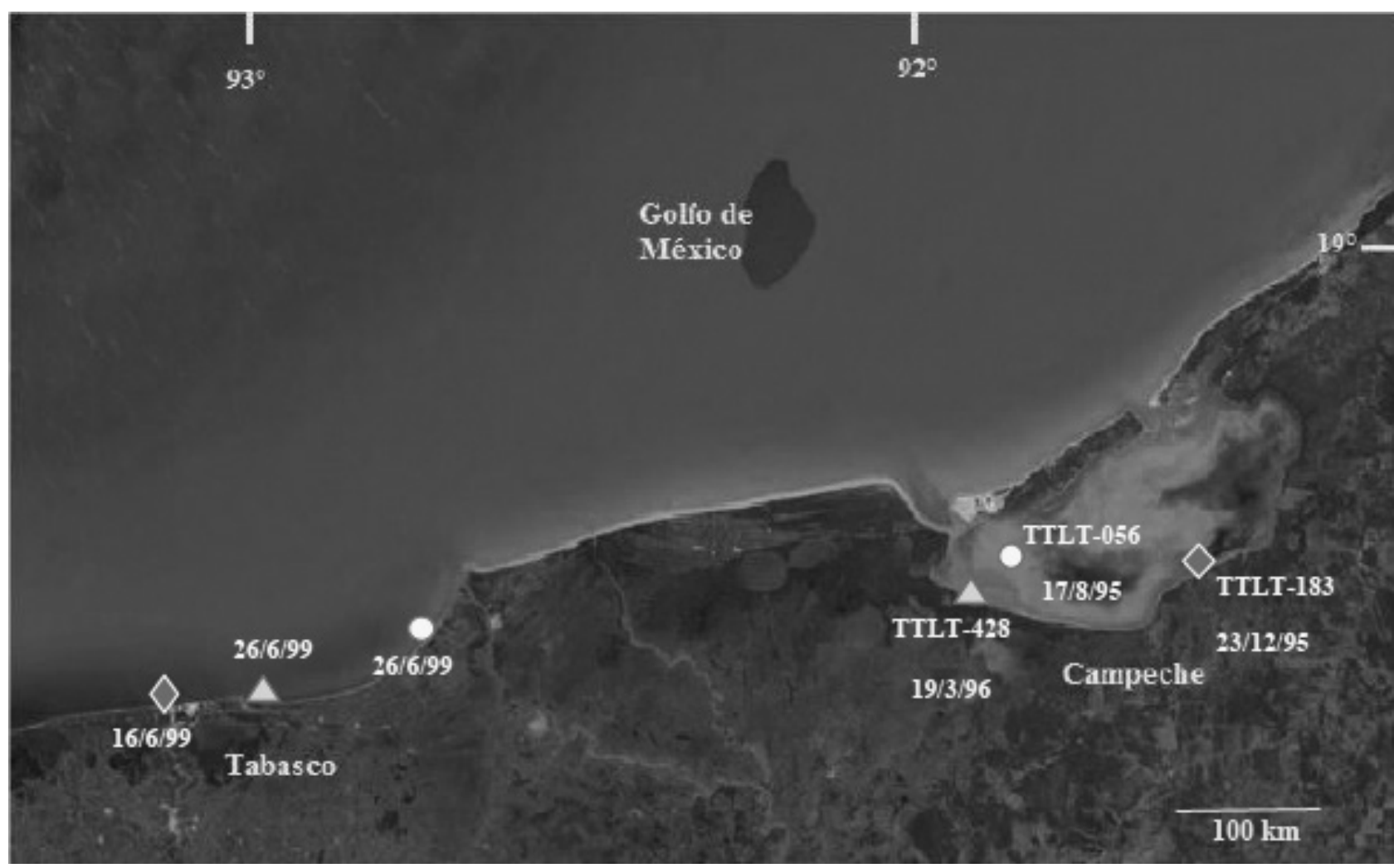

Figura 4. Movimientos realizados por las toninas identificadas por primera vez en la laguna de Términos y reavistadas años después en la costa de Tabasco (Tomado de Google Earth 15 abril, 2015, y modificado por el autor). 
además marcas en la aleta dorsal, por medio de las cuales pudo ser identificado de manera más continua. Este es otro ejemplo de las diferencias en la probabilidad de identificación que tienen los individuos, de acuerdo con su comportamiento y el tipo y posición de sus marcas.

Con respecto a catálogos de fotografías de zonas muy amplias como la costa del Atlántico de los Estados Unidos de Norteamérica (Urian et al. 1999), de 2,033 individuos identificados no se encontraron reavistamientos entre el norte (New Jersey) y el sur (Florida), indicando escaso movimiento entre estas áreas. El número de delfines en el catálogo del Atlántico medio es ligeramente menor que la estimación poblacional mínima de 2,482 toninas usada como stock para esta zona, sugiriendo que la estimación está sesgada negativamente.

En este estudio del sur del Golfo de México se trabajó en una franja de costa menor, ya que no se realizó esfuerzo en toda la costa sino en cuatro regiones particulares, el número de individuos fotoidentificados fue mayor que el registrado por Urian et al. (1999), por lo que quizá sea un indicativo de un tamaño poblacional mayor.

Existió un flujo mayor entre zonas cercanas, ya que cuatro individuos fueron localizados por primera vez en la laguna de Términos, en Campeche, y posteriormente entre 535 y 1,404 días después se registraron en la costa de Tabasco. Al parecer en la costa del Golfo de México la mayor parte de las toninas se están moviendo activamente a lo largo de la costa, o bien entrando y saliendo de ella, y algunos individuos utilizan zonas más restringidas para realizar sus actividades y se dispersan menos.

Quizá el caso más interesante de estas cuatro toninas lo constituye el individuo TTLT-056, quién se observó durante agosto y noviembre de 1995 y julio de 1996 en la laguna de Términos, para después registrarse el 26 de junio de 1999 en la costa de Tabasco (Figura 4), con el máximo registro de movimiento entre localidades de $320 \mathrm{~km}$, en 1,076 días. Durante su estancia en la laguna de Términos ya se había registrado su gran vagilidad, ya que fue localizado en ambas bocas de la laguna, lo cual significa viajes aproximadamente de entre 55 y $70 \mathrm{~km}$.

Es probable que este tipo de viajes los realicen la mayoría de las toninas en su búsqueda de alimento y zonas de reproducción y protección contra depredadores; pero no se había detectado anteriormente debido a la carencia de información y esfuerzo fotográfico, además de la escasa o nula coordinación e intercambio de información entre investigadores. No sería nada raro que si se revisaran los registros fotográficos de aletas dorsales de toninas de zonas como Veracruz, se llegaran a encontrar individuos previamente identificados en regiones sureñas del Golfo de México.

Con respecto a los movimientos realizados por las toninas, los datos más confiables han sido los obtenidos cuando por diversos motivos se instrumentan individuos con aparatos de emisión y recepción de satélite, los cuales proporcionan información prácticamente instantánea o en intervalos de tiempo muy cortos, de tal forma que prácticamente se puede seguir a un individuo hora a hora. Por ejemplo, la zona que más oportunidades han brindado para realizar este tipo de estudios es la costa de Florida en los Estados Unidos, ya que se han instrumentado toninas rehabilitadas después de haber varado, o bien proyectos específicos de marcaje para obtener este tipo de información.

Entre los primeros estudios realizados con toninas colocándoles aparatos de satélite está el de Tanaka (1987), el cual instrumentó seis machos y ocho hembras de toninas en aguas de Japón registrando que el animal que más viajó fue una hembra, la cual recorrió $603.9 \mathrm{~km}$, a una velocidad promedio de $1.4 \mathrm{~km} / \mathrm{h}$; en segundo lugar estuvo un macho que se movió $419.1 \mathrm{~km}$, a una velocidad promedio de $2.7 \mathrm{~km} / \mathrm{h}$, mientras que otra hembra recorrió $298 \mathrm{~km}$ a una velocidad de $2.1 \mathrm{~km} / \mathrm{h}$.

Mate et al. (1995) instrumentaron a una hembra de tonina, acompañada por su cría de cinco años de edad. Se siguieron sus movimientos durante 25 días. Los dos primeros días después de 
la captura y marcaje, el animal se movió en la "vieja" Tampa Bay. La tonina se localizó en la misma área entre el 7 y el 10 de julio de 1990 y no se volvió a ver hasta el 20 de noviembre del mismo año, 114 días después de que se recibió la última transmisión. Durante 25 días de registro la tonina viajó al menos $581 \mathrm{~km}$, con una velocidad mínima de $1.2 \pm 0.1 \mathrm{~km} / \mathrm{h}$. La máxima velocidad registrada fue de $4.9 \mathrm{~km} / \mathrm{h}$ durante 1.6 horas. La máxima distancia recorrida en un solo día fue de $50.2 \mathrm{~km}$. En promedio la tonina se movió $23.7 \pm 2.4$ km/día; este registro se puede considerar típico de una tonina residente de un sistema costero.

El registro más interesante y que proporcionó información de gran valor para el conocimiento de la especie fue el logrado por Wells et al. (1998), en el cual se obtuvieron datos de toninas pelágicas rehabilitadas después de haber varado y que posteriormente fueron liberadas con aparatos de satélite para registrar sus movimientos. Un macho de $270 \mathrm{~cm}$ de longitud total varado en la costa de Florida se liberó y poco más de un mes después se dejó de recibir su señal, indicando que se movió al menos 2,050 km, desde la punta sur de Florida hasta la parte norte de Cabo Hatteras en Carolina del Norte. Por otro lado, se instrumentó otro macho de $289 \mathrm{~cm}$ de longitud varado también en Florida, se liberó en ese mismo estado y se tuvo una recepción continua durante 40 días, localizándose por última vez $590 \mathrm{~km}$ al noreste de las islas Vírgenes, indicando que viajó más de 3,860 km desde su liberación. Después de la liberación se movió a lo largo de la costa en aguas de 20 a $30 \mathrm{~m}$ de profundidad; en su viaje a las islas caribeñas atravesó aguas de más de 5,000 m de profundidad, moviéndose contra la corriente ecuatorial.

Los resultados de movimientos obtenidos en este trabajo, son los primeros registros de movimientos desde la parte noreste de la península de Yucatán hasta la costa de Tabasco, es el más largo registrado hasta ahora en México, aunque como ya se señaló, quizá más adelante y bajo un esquema interinstitucional, o bien realizando esfuerzo de fotoidentificación en otros estados, se puedan encontrar movimientos aún más largos en todo el Golfo de México.

Con respecto a los movimientos de Campeche a Tabasco, al parecer son distancias que fácilmente pueden recorrer las toninas en búsqueda de condiciones más favorables pero, sobre todo, se ha observado que los machos son los que tienden a realizar estos viajes con el fin de encontrar grupos de hembras con las cuales puedan interactuar, conformando de este modo un vector muy importante para el flujo genético (Caballero et al. 2011). Al respecto, Wells et al. (1987) señalan que aparentemente los machos maduros desarrollan dos patrones diferentes de relaciones con las hembras adultas. Los machos residentes permanecen en áreas frecuentadas por gran número de hembras y se asocian con muchas de ellas, y hay grupos de machos "vagabundos" que tienden a moverse en grandes áreas y se asocian con grupos pequeños de hembras adultas en cualquier momento.

Würsig et al. (2000) señalan que se ha demostrado que algunas especies de cetáceos de aguas templadas (por ejemplo las toninas de la costa este de Estados Unidos), pueden tener una migración parcial, ya que algunos miembros se mueven a diferentes áreas durante una temporada y otros permanecen confinados toda su vida cerca de la costa. Los factores que determinan las migraciones parciales no son conocidos, se ha observado que pueden involucrar varias poblaciones parcialmente solapadas, explotando diferentes presas.

De acuerdo con la evidencia fotográfica, en la costa de Tabasco y en la zona de Celestún, Yucatán, no se han establecido patrones de residencia permanente $y$, al parecer, las toninas en Tabasco se mueven mucho a lo largo de la costa, por lo que pueden considerarse como visitantes estacionales u ocasionales, o bien la otra opción es que sean residentes pero en una zona mucho más amplia de la que abarcaron las zonas de estudio. Un factor en común entre estas dos áreas es que los sistemas costeros asociados no presentan condiciones favorables para el establecimiento de grupos de forma permanente. 
Otra evidencia de que las condiciones óptimas para el mantenimiento de un gran número de individuos son muy puntuales en espacio y tiempo, es la presencia de grupos de delfines de dientes rugosos (Steno bredanensis) cerca de la costa en Tabasco, entre 1992 y 1999. De manera invariable, estas numerosas manadas de estenos siempre han estado asociadas en eventos de alimentación, mezcladas con grupos de toninas en los meses de secas y lluvias (Delgado 1994; López y Delgado 2000).

Por otro lado, sucede lo contrario en las lagunas de Términos y Yalahau, en donde las condiciones son propicias para que se establezcan de manera permanente grupos de toninas a lo largo del año y durante varios años. Las características del hábitat lagunar, entre las que se cuentan la alta productividad y protección contra condiciones atmosféricas adversas como huracanes y marejadas, permiten que las toninas puedan cubrir todos sus requerimientos a lo largo del año, por lo que prácticamente no tendrían motivos para moverse a otras zonas, ya que la variedad de microhábitats les proporciona alimento durante todo el año.

De acuerdo con las características de cada laguna en cuanto a dimensiones y valores de productividad, se tiene que en la laguna de Términos, Campeche, hay mayores opciones para mantener a una población residente más numerosa, mientras que en Yalahau el número de toninas que pueden permanecer durante largos periodos es mucho menor, y esto se refleja en el número de toninas registradas. Por estas mismas características, estas lagunas son frecuentadas de manera estacional por cientos de toninas que permanecen poco tiempo en sus alrededores, por lo que se presentan todas las posibles variedades de residencia, es decir: anual, multinanual, temporal y ocasional. Cabe señalar el caso particular del "Mocho" (TTLT-001), siendo el animal que tiene un registro más largo en la laguna de Términos, con 10 años nueve meses de permanencia, aunque no se descarta la posibilidad de que este individuo haya salido de la laguna al Golfo de México.

En el caso de los residentes temporales, llegan a finales de la temporada de secas y permanecen durante las lluvias en ambas lagunas, sacando provecho de la abundancia de alimento. Por otro lado, hay hembras preñadas que comienzan a llegar a la laguna de Términos en el mes de febrero y permanecen allí durante dos o tres meses. Muchos de estos animales se quedan de manera permanente una vez que se familiarizan con el hábitat y aprenden a sacarle provecho a lo largo del año.

Podría decirse desde, otro punto de vista, que quizá en la laguna de Términos se presentan residentes anuales, pero que utilizan estacionalmente las diferentes zonas de la laguna según sus necesidades, realizando pequeñas migraciones locales, además siempre existe la opción de salir hacia el Golfo de México en caso de que en el interior de la laguna se presente algún problema como la presencia excesiva de actividad humana.

\section{Conclusiones}

La distribución de avistamientos en las zonas costeras de Tabasco y Yucatán estuvo asociada con la presencia de desembocaduras de sistemas fluviales, mientras que en las lagunas costeras las toninas prefirieron estar en el interior de las cuencas, sobre todo en la laguna de Términos, ya que en Yalahau la distribución fue similar dentro y fuera de la laguna; esto también tiene relación con las ventajas que ofrece cada sistema, como la protección contra eventos climáticos adversos y contra depredadores, abundancia de alimento así como áreas de crianza.

En la costa de Tabasco y en la costa frente a isla Holbox se registraron movimientos latitudinales con más de $40 \mathrm{~km}$ de diferencia. Al parecer los movimientos locales más largos se presentan en la laguna de Términos, $70 \mathrm{~km}$ para moverse de un extremo a otro. En general las toninas se pueden mover de 5 a $40 \mathrm{~km}$ en un solo día, dependiendo de las actividades que realicen. 
Se registraron cinco movimientos de individuos a largo plazo, tanto en espacio como en tiempo, siendo el más largo de 247 días, al menos $800 \mathrm{~km}$. Estos movimientos pueden ser indicio de un continuo poblacional en las toninas costeras del sur del Golfo de México.

Los datos mostraron diferentes grados de fidelidad al sitio, siendo la laguna de Términos, Campeche, la que presenta mayores grados de residencia. Además de presentarse patrones de residencia anual y multianual, en la mayoría de las zonas las toninas tienden a pasar periodos cortos y medios en las diferentes localidades, conformando residencias estacionales, o bien son visitantes ocasionales. Puede ser que el ámbito hogareño de los grupos registrados sea mayor al definido como zona de estudio.

\section{Agradecimientos}

A lo largo de los años de trabajo de campo siempre se contó con el apoyo de varios compañeros y colegas; se gradece su valiosa ayuda a: J. G. Ortega Ortiz, L. E. Vázquez Maldonado, I. López Hernández, A. Romero Tenorio, V. Islas Villanueva, I. Reza García, I. Segura, así como a otros estudiantes de biología. A los pescadores de los poblados de isla Aguada, Campeche, isla Holbox, Quintana Roo, Chiltepec y Puerto Ceiba, Tabasco y Celestún, Yucatán, principalmente a S. García por guiarme en los recorridos en la laguna de Términos. A la empresa Via Delphi, que brindó su apoyo durante la realización de los trabajos de campo. AI CONACyT y a la Dirección General de Estudios de Posgrado de la UNAM, por el apoyo otorgado.

Se contó con permisos de investigación para la realización de este estudio, otorgados por diversas instituciones: Permiso 2775 de la Secretaría de Pesca (1994), 408/94 Secretaría de Marina, 2652 SEMARNAP (1995), 364 INE (1995), D00700(2)3614 INE, 1356 SEMARNAP (1996), 1356 SEMARNAP (1997), 1104 SEMARNAP (1998) y 1089 SEMARNAP (1999).

\section{Resumen}

Introducción: Se sabe poco de los movimientos y patrones de residencia de las toninas en la parte sur del Golfo de México. El objetivo de este estudio es tener información sobre este tema, debido a que la laguna de Términos es la zona con mayor esfuerzo de estudio en la región, y es posible que las toninas se muevan a lo largo de la costa.

Material y métodos: Entre 1994 y 1999 se realizaron recorridos en el sureste del Golfo de México (isla Holbox, Quintana Roo, Celestún, Yucatán, laguna de Términos, Campeche y la costa de Tabasco), con el fin de registrar los movimientos y residencia de poblaciones de toninas.

Resultados: Utilizando la técnica de fotoidentificación se diferenciaron 2,889 individuos, siendo la laguna de Términos la que presentó la mayor cantidad $(1,987)$, registrando un individuo con una residencia multianual de 10.9 años. Otro individuo se observó durante 1997 en isla Holbox y 247 días después se le identificó en Tabasco. Otros cuatro individuos se movieron de la laguna de Términos, Campeche, a Tabasco, viajando en promedio $270 \mathrm{~km}$, constituyendo cronológicamente los primeros registros de residencia a largo plazo y de migración en el sur del Golfo de México.

Discusión y conclusiones: Se encontró que las toninas tienen áreas de uso preferencial como las lagunas costeras, en donde encuentran alimento y refugio para sus crías. El registro de los movimientos en zonas tan distantes como Quintana Roo y Tabasco puede ser un indicio de un continuo poblacional en las toninas costeras del sur del Golfo de México.

Palabras clave: Golfo de México; movimientos; residencia; Toninas; Tursiops truncatus.

\section{Literatura Citada}

BallanCE, L. 1990. Residence patterns, group organization, and surfacing association of bottlenose dolphins in Kino Bay, Gulf of California, Mexico. Pp. 267-283 en The bottlenose dolphin (Leatherwood, S., y R. R. Reeves, eds.). Academic Press. San Diego, EE.UU. 
Bazúa Durán, M.C. y A. Delgado-Estrella. 2014. Los tursiones, delfines de la laguna de Términos. Revista FOMIX-Campeche 19:20-26.

Bearzı, M. 1996. Bottlenose dolphins in El Palmar and Rio Lagartos reserves (Yucatan, Mexico): A preliminary study. Proceedings ACS. 8-11 November, San Pedro CA, EE. UU.

Bräger, S. B. Würsig, A. Acevedo and T. Henningsen. 1994. Association patterns of bottlenose dolphins (Tursiops truncatus) in Galveston Bay, Texas. Journal of Mammalogy 75:431-437.

Caballero, S., V. Islas-Villanueva, G. Tezanos-Pinto, S. Duchene, A. Delgado-Estrella, R. Sánchez-Okrucky And A. A. Mignucci-Giannoni. 2011. Phylogeography, genetic diversity and population structure of common Bottlenose Dolphins in the Wider Caribbean inferred from analyses of mitocondrial DNA control region sequences and microsatellite loco: conservation and management implications. Animal Conservation COMPLETAR

Darby, F. L., K. W. URian and R. S. Wells. 1995. Sex and age differences in dorsal fin natural markings of the bottlenose dolphin, Tursiops truncatus, from Sarasota Bay, FL. Pp. 28 en XI Biennial Conference on the Biology of Marine Mammals. Orlando, EE. UU.

Defran, R. H., G. M. Shultz, AND D. W. Weller. 1990. A technique for the photographic identification and cataloging of dorsal fin of the bottlenose dolphin (Tursiops truncatus). Report International Whaling Commission (Hammond, P. S., S. A. Mizroch, and G. P. Donovan). Special Issue 12. Cambridge, EE. UU.

De La Lanza Espino, G. 1991. Oceanografía de los mares mexicanos. AGT. Editores. Ciudad de México, México.

De La Parra Venegas, R. y B. E. Galván Pastoriza. 1985. Observación del tursión costero del Pacífico en el sistema Topolobampo-Ohuria, Sinaloa (con notas acerca del comportamiento, ritmo respiratorio e identificación individual). Pp:137-160 en X Reunión Internacional sobre Mamíferos Marinos. La Paz, México.

Delgado Estrella, A. 1991. Algunos aspectos de la ecología de poblaciones de las toninas Tursiops truncatus, Montagü 1821, en la laguna de Términos y Sonda de Campeche, México. Tesis de Licenciatura, Biología. ENEP Iztacala, UNAM. Ciudad de México, México..

Delgado-Estrella, A. 1994. Presencia del delfín de dientes rugosos o esteno (Steno bredanensis, Lesson 1828), en el estado de Tabasco, México. Anales Instituto de Biología Universidad Nacional Autónoma de México, Serie Zoología 65:303-305.

Delgado-Estrella, A., J. G. Ortega Ortiz, y A. Sánchez Ríos. 1994. Prospección poblacional de toninas Tursiops truncatus, en la laguna de Términos, Campeche, México. Informe no publicado para Via Delphi S.A. de C. V.

Delgado-Estrella, A., y H. Pérez-Cortés M. 1993. Abundancia y distribución temporal de toninas (Tursiops truncatus) en la costa del sur del Golfo de México. XVIII Reunión Internacional para el Estudio de los Mamíferos Marinos. La Paz, México.

Delgado Estrella, A. 2002. Comparación de parámetros poblaciones de las toninas, Tursiops truncatus, en las región sureste del Golfo de México (Estados de Tabasco, Campeche, Yucatán y Quintana Roo). Tesis doctorado en ciencias (Biología), Facultad de Ciencias, UNAM, México. Ciudad de México, México.

García, E. 1973. Modificaciones al sistema de clasificación climática de Köppen. Instituto de Geografía, UNAM. Ciudad de México, México.

Hammond, P. S., S. A. Mizroch, And G. P. Donovan. 1990. Report of the workshop on individual identification and the estimation of cetacean population parameters. Individual recognition of cetaceans: Use of photo-identification and other techniques to estimate population parameters. Pp. 3-17 en Report International Whaling Commission (Special Issue 12). Cambridge, Reyno Unido.

HeCKel, D. G. 1992. Fotoidentificación de tursiones, Tursiops truncatus (Montagü, 1821), en la boca de Cazones de la laguna de Tamiahua, Veracruz, México (Cetacea: Delphinidae). Tesis Profesional. Facultad de Ciencias, UNAM. Ciudad de México, México. 
Herrera-Silveira, J. A. 1990. Productividad primaria fitoplanctónica de la laguna de Celestúm. Pp. 91 en II Congreso Ciencias del Mar.

Lechuga-M. A., A. Salinas-Z., D. Castillo-L., And C. Álvarez. 1995. Bottlenose dolphin group structure and habitat use around Holbox island, Q. Roo, Mexico. Pp. 67 en XI Biennial Conference on the Biology of Marine Mammals.14-18 December. Orlando EE.UU.

Longhurst, A. 1998. Ecological geography of the sea. Academic Press. San Diego, EE.UU.

López Hernández, I. 1997. Ecología poblacional de las toninas Tursiops truncatus en la costa de Tabasco, México. Tesis Licenciatura Biología, Facultad de Ciencias, UNAM. Ciudad de México, México.

López-Hernández, I., y A. Delgado-Estrella. 2000. Observaciones sobre grupos de delfines de dientes rugosos, Steno bredanensis, en la costa de Tabasco, México. Pp. 47 en XXV Reunión Internacional para el Estudio de los Mamíferos Marinos. La Paz, México.

Mate, B. R., K. A. Rossbach, S. L. Nieukirk, R. S. Wells, A. B. Irvine, M. D. Scott, and A. J. Read. 1995. Satellitemonitored movements and dive behavior of a bottlenose dolphin (Tursiops truncatus) in Tampa Bay, Florida. Marine Mammal Science 11:452-463.

Mollinari, R. L., AND J. Morrison. 1988. The separation of Yucatan current from the Campeche Bank and the penetration of the Loop current into the Gulf of Mexico. Journal Geophysical Research 93:1064510654.

Morteo, E., A. Rocha-olivares, and L. G. Abarca-Arenas. 2014. Sexual segregation in coastal bottlenose dolphins (Tursiops truncatus) in the south-western Gulf of Mexico. Aquatic Mammals 40:375-385.

Ortega-Ortiz, J. G., Y A. Delgado-Estrella. 1996. Abundancia de toninas Tursiops truncatus en la Bahía de Agiabampo (Sonora-Sinaloa) durante julio de 1995. Resúmenes de la XXI Reunión Internacional para el Estudio de los Mamíferos Marinos SOMEMMA. Chetumal, México.

Paluskiewicz, T., L. P. Atkinson, E. S. Posmentier, and C. R. McClain. 1983. Observation of a loop current frontal eddie intrusion onto the west Florida continental shelf. Journal Geophysics Research 88:9639-9651.

Shane, S. H., R. S. Wells, and B. Würsig. 1986. Ecology, behavior and social organization of the Bottlenose Dolphin: A review. Marine Mammal Science 2:34-63.

TANAKA, S. 1987. Satellite radio tracking of bottlenose dolphins, Tursiops truncatus. Nippon Suisan Gakkaishi 53:1327-1338.

Urian, K., S. Baker, S. Barco, N. Bowles, M. Caldwell, C. Gubbins, L. Hansen, A. Hohn, R. Mallon-Day, T. Murphy, R. Petricig, A. Read, K. Rittmaster, G. Rountree, W. Ryan, L. Sayigh, D. Schofield, J. Tapia, V. Thayer, and E. Zolman. 1999. The Mid-Atlantic bottlenose dolphin photo-id catalog: A cooperative approach to examine stock structure. Pp. 191 en Proceedings of $13^{\text {th }}$ Biennial Conference on the Biology of Marine Mammals. Wailea, EE. UU.

Walsh, J. J., D. A Dieterle, M. B. Meyers, and F. E. Müller-Karger. 1989. Nitrogen exchange at the continental margin: A numerical study of the Gulf of Mexico. Prog. Ocenaogr. 23, 245-301.

Wells, R. S., M. D. Scott, ANd A. B. Irvine. 1987. The social structure of free-ranging bottlenose dolphins. Pp. 247-305 en Current Mammalogy (Genoways, H. H. ed.)., Vol. I. Plenum Press. New York, EE. UU.

Wells, R. S., L. J. Hansen, A. L. Baldrich, T. P. Dohl, D. L. Kelly, and R. H. Defran. 1990. Northward extension of the range of bottlenose dolphins along the California coast. Pp. 421-431 en The bottlenose dolphin (Leatherwood S., and R. R. Reeves, eds.). Academic Press. San Diego, EE. UU.

Wells, R. S., A. J. Westgate, H. L. Rhinehart, P. Cunningham, J. Whaley, D. P. Costa, A. J. Read, T. Hempburn, M. Baran, C. Koberna, AND T. Cox. 1998. Gulliver's travels: First record of long distance movements of offshore bottlenose dolphins. Pp. 147 en The World Marine Mammal Science Conference. Monaco, Monaco.

Würsig, B., AND T. A. Jefferson. 1990. Methods of photo-identification for small cetaceans. Pp. 43-52 en Report International Whale Commission (Hammond, P. S., S. A. Mizroch, and G. P. Donovan, eds). Special Issue 12. Cambridge, EE. UU.

Würsig, B., T. A. Jefferson, And D. J. Schidly. 2000. The marine mammals of the Gulf of Mexico. Texas A \& M University Press. College Station, EE. UU. 
Yáñez-Arancibia, A., A. L. Lara-Domínguez, P. Sánchez-Gil, y H. Álavarez-Guillén. 1988. Evaluación ecológica de las comunidades de peces en la Laguna de Términos y Sonda de Campeche. Contribución 556 del ICMyL, UNAM. Pp. 323-355 en Ecología de los ecosistemas costeros en el sur del Golfo de México: La región de la Laguna de Términos. 1988. UNAM \& FAO. Distrito Federal, México

Zacarías A, J., Y E. ZÁrate B. 1992. Primeras contribuciones a la ecología de Tursiops truncatus en las costas de Quintana Roo, México, durante 1986 a 1989. Resúmenes XVII Reunión Internacional para el Estudio de los Mamíferos Marinos, 22-25 abril 1992. La Paz, México.

Summited: February 5, 2014

Review: March 25, 2015

Accepted: April 12, 2015

Associated editor: Juan Pablo Gallo 